\title{
CULTURA DEL EMPRENDIMIENTO EN INSTITUCIONES DE EDUCACIÓN SUPERIOR: ESTRATEGIA DE INCLUSIÓN SOCIAL. ${ }^{1}$
}

\author{
CULTURE OF ENTREPRENEURSHIP IN HIGHER EDUCATION INSTITUTIONS: SOCIAL INCLUSION \\ STRATEGY.
}

\author{
Zambrano Valdivieso Oscar ${ }^{2}$ Espitia González Sandra ${ }^{3}$ \& Hernández Ardila Josée \\ Corporación Universitaria Minuto de Dios
}

RECIBIDO: Agosto 06 de 2016

ACEPTADO: Noviembre 13 de 2016

\begin{abstract}
RESUMEN
La presente investigación tuvo como objetivo determinar la importancia de la cultura del emprendimiento en las Instituciones de Educación Superior como estrategia de inclusión social; metodológicamente, el estudio fue de tipo exploratorio y descriptivo, con enfoque cualitativo y un método deductivo a partir del análisis documental recolectado. Se empleó como instrumento de recolección de la información secundaria la ficha de registro de datos. Los resultados demostraron que, si bien existe una normatividad expresa y claramente definida sobre la aplicación de la cultura del emprendimiento, aun no es clara su aplicabilidad como estrategia de inclusión social en las Instituciones de Educación Superior (IES). En conclusión, las IES deben revaluar sus Proyectos Educativos Institucionales (PEIs), Programa Curricular de los Programas (PCPs) y esquemas curriculares en donde se involucre el emprendimiento, ya que son realmente pocos los esfuerzos y escasos los éxitos que sobre el tema pueden analizarse. Se recomienda continuar con el seguimiento a los procesos que la normatividad ha permitido iniciar para lograr que la cultura del emprendimiento en las IES se convierta en una estrategia real de inclusión social.
\end{abstract}

Palabras clave: Cultura, emprendimiento, estrategia, IES, inclusión social.

\section{ABSTRACT}

The present research aims to determine the importance of entrepreneurship culture in Higher Education Institutions as a strategy of social inclusion. Methodologically, the study is exploratory and descriptive, with a qualitative approach and a deductive method based on a collected documentary analysis. The data log sheet was used as an instrument for collecting the secondary information. The results showed, that although there is an express and clearly defined regulation on the application of entrepreneurship culture, its applicability as a strategy for social inclusion in HEIs (Higher Educational Institutions) is not yet clear. In conclusion, HEIs should reevaluate their PEIs (Institutional Program of Education), their PCPs (Curricular Programs) and their curricular schemes, where entrepreneurship is involved, since there are really few efforts and few successes that can be analyzed on the subject. It is recommended to continue with the surveillance of the processes that the legislation has allowed to start in order to let the culture of entrepreneurship in HEIs be a real strategy for social inclusion.

Keyword: Culture, entrepreneurship, IES, strategy, social inclusion.

\section{Este artículo se puede referenciar}

Zambrano, O.; Espitia, S. \& Hernández, J. (2017). Cultura del emprendimiento en Instituciones de Educación Superior: estrategia de inclusión social. En Desarrollo Gerencial Revista de la Facultad de Ciencias Económicas Administrativas y Contables de la Universidad Simón Bolívar-Colombia, 9(1), 176-191.

\footnotetext{
${ }^{1}$ Artículo de revisión.

${ }^{2}$ Profesor investigador. Grupo de Investigación GIDTI-UNIMINUTO y GECOA-UNICIENCIA. Cra. 19 No. 36-75 Of. 303.

zambranoscar@gmail.com.3003007683.Bucaramanga, Colombia.

${ }^{3}$ Profesor Ciencias Empresariales Uniminuto. Samaesgo13@hotmail.com. 3004494621. Bucaramanga, Colombia.

${ }^{4}$ Profesor Ciencias Empresariales Uniminuto.jomisaelh@hotmail.com. 3108125079. Bucaramanga, Colombia.
} 


\section{1.- INTRODUCCIÓN}

En las últimas dos décadas, se ha evidenciado en el ambiente académico y empresarial, el auge del emprendimiento, con la participación de instituciones públicas, privadas y sociales. El surgimiento de este campo de conocimiento se inscribe dentro de la disciplina de la administración y puede atribuirse a varios fenómenos: de un lado, a la velocidad de los cambios tecnológicos, a los procesos de la globalización, y de otro, a la necesidad de revivir el emprendimiento empresarial que ha caracterizado la humanidad, enriqueciendo el mundo y la cultura y ha sido importante motor del progreso humano (Orrego, 2009). Por su parte, Rodríguez (2009, citado por Durán, Parra y Márceles 2015) expresan que el emprendimiento hay que entenderlo e interpretarlo desde diversas perspectivas, para estudiar su complejidad, construyendo un discurso inter y transdisciplinario que establezca nuevas formas para su consulta, estudio y entendimiento.

En este orden de ideas, Durán, Fuenmayor, Cárdenas y Hernández (2016) y Redondo, Pertúz y Vidal (2015) plantean que el emprendimiento es la capacidad de llevar a cabo proyectos de acción en el marco de un contexto de oportunidades identificadas y que contiene la suficiente energía para organizar los medios y recursos para aplicarlo. Por lo que el estímulo a la actividad emprendedora gira en torno a las políticas de desarrollo económico nacional.

Frente a esta situación, la dinámica del emprendimiento ha incursionado en las Instituciones de Educación Superior (IES) como artífice de consolidación en el proceso de enseñanza-aprendizaje, como lo demuestran Tarapuez, Osorio \& Botero (2013), surge en Colombia la Ley 1014 de 2006 (Congreso de la República de Colombia, 2006) más conocida como Ley de Emprendimiento que a pesar de lo general de su marco jurídico, generó instrumentos adicionales para promover el emprendimiento y creó un ambiente propicio para incluir la creación de nuevas empresas como un tema específico que debe ser coordinado por el Estado en unión con una variedad de instituciones públicas y privadas que de una u otra manera tienen que ver con la cadena de valor del sector productivo y académico.

En el entorno socioeconómico, para Ruiz \& Duarte (2009), el empleo y la educación son derechos fundamentales para los ciudadanos en los estados sociales, estos son garantía para generar condiciones de vida dignas y facilitar el desarrollo económico y social del país, en circunstancias de equidad y de bienestar general, ofreciendo oportunidades que orienten y propicien el desarrollo humano; pero, para que el emprendimiento genere no solo empleo e ingresos sino también innovación, se requiere la convergencia e integración "cuatripartita” entre Universidad, Estado, Comunidad y Empresa, como lo refuerzan Ramos \& Bayter (2012).

Con este principio de convergencia hacia lo social, desde la óptica cuatripartita, la transformación hacia la sociedad inclusiva será posible a través del compromiso y cooperación de los gobiernos en sus diferentes niveles, la comunidad y sus instituciones, la fuerza y experiencias de las empresas con la 
academia, es decir, con la responsabilidad social de las IES como líder de esta fuerte misión. Frente a estos retos es importante determinar ¿cómo el emprendimiento se convierte en una estrategia de inclusión social en las Instituciones de Educación Superior?

En función del objetivo propuesto y la tipología de investigación, la presente investigación es en primer término de tipo exploratorio, considerando el carácter bibliográfico empleado mediante el análisis de literatura específica sobre el tema del escrito y en segundo término de tipo descriptivo con el análisis de Instituciones de Educación superior colombianas con orientación en emprendimiento de carácter regional y nacional, con un enfoque cualitativo participativo y un método deductivo a partir del análisis documental recolectado.

Respecto a la población para este trabajo se estudiaron 5 instituciones de educación superior que cuentan con unidad de emprendimiento reconocida por el Fondo Emprender - SENA en el Área Metropolitana de Bucaramanga, Santander (Colombia), empleando como instrumento de recolección de la información secundaria la ficha de registro de datos aplicada a libros, boletines, revistas, folletos, y periódicos que se utilizaron como fuentes para recolectar datos sobre las categorías de interés. Como alcance metodológico, inicialmente, se detectó el problema de estudio a fin de definir los objetivos que permitieron solucionar dicha problemática. Luego se seleccionaron las teorías para sustentar el estudio con base en la categoría de estudio. Ya una vez establecida la metodología, se diseñó el instrumento de recolección de datos para su posterior aplicación para finalmente generar los resultados y conclusiones presentadas en este artículo.

El emprendimiento se ha posicionado al interior de las universidades como uno de los temas bandera y de interés general, por considerar que la formación disciplinar no es suficiente para desempeñarse exitosamente en el mundo actual; es necesario además, formar capacidades, fortalecer competencias y ofrecer alternativas de vida para los futuros profesionales acorde con lo afirmado por el Ministerio de Comercio, Industria y Turismo, en el marco de un convenio firmado con la Asociación Colombiana de Universidades - ASCUN (2014).

Es desde esta entidad, que se tiene como propósito aunar esfuerzos técnicos y financieros, para apoyar el fortalecimiento del emprendimiento universitario con actividades extracurriculares, así como el acompañamiento al desarrollo de políticas institucionales, que respaldan el desarrollo del ecosistema del emprendimiento al interior de las IES. Para las Instituciones de Educación Superior del país, está claro que el desarrollo del emprendimiento y la innovación, va ligado de las políticas y el respaldo que otorgan dichas organizaciones a la comunidad y a los actores o stakeholders involucrados, que los lleve a lograr un empoderamiento en emprendimiento e innovación. 
En el estudio de la Organización Internacional del Trabajo (OIT), (Tendencias del empleo mundial para los jóvenes, 2004), se reveló, que los jóvenes representan el 25 por ciento de la población en edad laboral (de 15 a 64 años), pero conforman el 47 por ciento del total de los 186 millones de desempleados en todo el mundo en el 2003. En el mismo sentido, un estudio de la OCDE (2001), concluyó que los jóvenes, constituyen uno de los grupos con tasas de desempleo más alta con relación al promedio de la población económicamente activa en muchas economías desarrolladas y en desarrollo.

Así, formarlos para la creación de nuevas empresas, implica apoyar la creación de oportunidades laborales que permita posiblemente fortalecer la estrategia de inclusión social establecida por las instituciones de educación superior ya que como perspectiva, en palabras de Blanco (2006) la inclusión demanda avanzar hacia un único sistema educativo que sea más diversificado para atender de forma adecuada las necesidades los distintos colectivos y personas, con el fin de lograr, por distintas vías equivalentes en calidad, aprendizajes equiparables para toda la población. Esto supone superar la actual separación entre la educación para todos y la educación para aquellos que por diferentes razones son considerados "diferentes".

\section{2.- MÉTODO}

A continuación, se presenta el método utilizado en el desarrollo de esta investigación.

\section{$\checkmark$ Diseño}

En función del objetivo propuesto para este trabajo y considerando la tipología de investigación como el grado de profundidad con el cual se pretende abordar el fenómeno, el mismo se considera de tipo exploratorio y descriptivo. El primero es de carácter bibliográfico mediante el análisis de literatura específica sobre el tema del escrito. El estudio descriptivo incluyó como unidad de análisis a 5 Instituciones de Educación superior colombianas con orientación en emprendimiento de carácter regional y nacional, con un enfoque cualitativo participativo y un método deductivo a partir del análisis documental recolectado que generó un análisis holístico y no individual del tema de estudio.

\section{$\checkmark$ Participantes}

Respecto a la población o conjunto de elementos, seres o eventos sobre los cuales se desea obtener información, para este trabajo se consideraron 5 instituciones de educación superior que cuentan con unidad de emprendimiento reconocida por el Fondo Emprender-SENA en el Área Metropolitana de Bucaramanga, Santander (Colombia). 


\section{$\checkmark$ Instrumentos}

En la presente investigación se empleó como instrumento de recolección de la información secundaria la ficha de registro de datos aplicada a libros, boletines, revistas, folletos, y periódicos que se utilizaron como fuentes para recolectar datos sobre las categorías de interés; este instrumento, permitió el registro e identificación de las fuentes de información, así como el acopio de datos o evidencias. La ficha de registro documental se construyó con base en el trabajo de carácter intelectual del equipo investigador, mediante el cual se manifiesta la capacidad de análisis y de devolución creativa. Mediante la lectura reflexiva y minuciosa se obtuvo la información requerida y útil tanto para la formación del marco teórico, sustentación del problema de investigación y desarrollo de objetivos.

\section{$\checkmark$ Procedimientos}

Inicialmente, se detectó el problema de estudio a fin de definir los objetivos que permitieran solucionar dicha problemática. Luego se seleccionaron las teorías para sustentar el estudio con base en la categoría de estudio. Ya una vez establecida la metodología, se diseñó el instrumento de recolección de datos para su posterior aplicación. Tras aplicar el instrumento en el análisis documental, se tabularon y analizaron los datos para finalmente generar los resultados y conclusiones presentadas en este artículo.

\section{3.- RESULTADOS}

Hoy, las instituciones deben apoyar la creación de puestos de trabajo basados en la innovación o el emprendimiento, para lograr que el país tenga organizaciones bien estructuradas, que aprovechen los recursos que posee y lo conduzcan hacia un posicionamiento internacional.

Las teorías asociadas al emprendimiento y la innovación datan de los últimos años del siglo XIX con Schumpeter, al determinar, que la función del emprendedor es determinante en los procesos de innovación y de inversión, como mecanismos para la creación de iniciativas en el campo económico, que generen valor, ya sea mediante la producción de nuevos productos, nuevos procesos de producción, nuevas formas de organización y/o nuevos mercados, (Camacho, 2007). De acuerdo con Orrego (2009), se debe considerar que de conformidad con la mirada mercantil de la economía, es común encontrar en el pensamiento tradicional schumpeteriano, la identificación del emprendedor con el sujeto comercial, que articula innovadoramente los distintos factores de producción, tomando algunos riesgos; sin desconocer la importancia de éste pensamiento objetivista, la noción a tener en cuenta en éste artículo, es precisamente aquélla que propone revelar la significación del emprendimiento desde la subjetividad.

A partir de esta premisa, las empresas no quieren correr riesgos al incluir en su nómina talento humano que no le apueste a la innovación y el emprendimiento; existen opciones mediante las cuales el gobierno 
apoya en conjunto con otras organizaciones al sector empresarial para lograr obtener innovaciones que mejoren la competitividad y este fin es precisamente el que se debe reforzar desde el interior de las organizaciones para generar procesos de mejora continua que conlleven a emprendimientos, intraemprendimientos y emprendedurismos dinamizando el entorno del personal, como de las instituciones.

En los últimos años, como afirma Camacho (2007), el gobierno y el sistema educativo en general, están promoviendo en las regiones, la cultura emprendedora, asumiendo la empresarialidad como una estrategia prioritaria, para adquirir una mayor conciencia y claridad sobre la importancia de la promoción del espíritu empresarial, la formación de empresarios y la creación de empresas, pero, no se debe quedar solo en generar ideas y esperar si se vuelven realidades al formalizar las empresas en la práctica, se debe generar todo un programa de acompañamiento que no se limite solamente a la aplicación del PHVA.

En palabras de Reinoso (2008); la ley 1014 de 2006 de fomento a la cultura del emprendimiento en Colombia, trata de la importancia de la formación empresarial en los currículos técnicos y tecnológicos para promover el espíritu emprendedor, entendido como la capacidad de generar bienes y servicios de una forma creativa, metódica, ética, responsable y efectiva, para generar un resultado que es la creación de valor que beneficia a la empresa, la economía y la sociedad. Como se aprecia, la ley referenciada permite la creación de redes nacionales y regionales de emprendimiento para integrar los actores que dinamizan la competitividad mediante emprendimientos innovadores; igualmente se establece su enseñanza de carácter obligatorio en todos los establecimientos oficiales y privados que ofrezcan educación formal en todos los niveles (preescolar, básica primaria, básica secundaria y media); pero, a la vez diseñando una estrategia curricular sobre emprendimiento y la generación de empresas.

Existe en Colombia el Fondo emprender, instituto creado mediante ley 789 de diciembre 27 de 2.002, adscrito al Servicio Nacional de Aprendizaje; es un Fondo, creado por el Gobierno Nacional de Colombia para financiar proyectos empresariales provenientes de Aprendices, Practicantes Universitarios (que se encuentren en el último año de la carrera profesional) o Profesionales que no superen dos años de haber recibido su primer título profesional. El objetivo del fondo es apoyar proyectos productivos que integren los conocimientos adquiridos por los emprendedores en sus procesos de formación, con el desarrollo de nuevas empresas.

Ahora, como resultado del informe: Estado del arte sobre emprendimiento universitario, desarrollado por el Ministerio de Comercio, Industria y Turismo y la Asociación Colombiana de Universidades ASCUN en el 2014; se aprecian experiencias como la de Toca (2010), quien desarrolla un estudio que tiene como antecedente la intención de crear el Centro de Emprendimiento de la Universidad del Rosario. $\mathrm{Su}$ propósito es dar luces sobre el concepto de emprendimiento; afirmar el carácter transversal y 
transdisciplinario del tema y reflexionar sobre las opciones que hay en la formación de emprendedores. Esto a partir de una revisión de bibliografía especializada, de experiencias en el campo de formación de emprendedores y de estudios sobre la medición del emprendimiento.

Jaramillo (2013) hace un estudio sobre la forma en que los individuos emprendedores deben ser formados a la luz de la didáctica, ya que considera que la enseñanza del emprendimiento, es un proceso educativo empírico que debe apoyarse en la pedagogía, para poder preparar estudiantes de un modo sistémico y eficiente. Igualmente, en el informe se destaca, que, dentro de las investigaciones dirigidas a estudiar la docencia en emprendimiento, está la realizada por la Red de Emprendimiento Universitario REUNE, durante los años 2011 y 2012. Como parte de los resultados de este estudio, Cubillos y Rodríguez (2013) proponen, que el docente tiene una gran responsabilidad por la labor que realiza, en la formación de los estudiantes universitarios. La docencia en emprendimiento, debe estar orientada hacia la formación en competencias, que le permitan al estudiante identificar oportunidades y proponer soluciones a los problemas de su entorno, desde el conocimiento de su disciplina o profesión; así mismo tomar decisiones, actuar, liderar y correr riesgos controlados.

Por último, es de resaltar, que en el informe del Ministerio de Comercio, Industria y Turismo y Asociación Colombiana de Universidades -ASCUN (2014), el emprendimiento en las instituciones de educación superior, es un tema que ha generado tanto interés y expectativas, debido a su aceptada participación en el crecimiento económico de una región o país. Efectivamente, tanto la educación en emprendimiento como el emprendimiento académico se han tratado de manera amplia; pero, Dickson, Solomon, \& Weaver (2008), también afirman que, en el estudio del emprendimiento, una variable institucional que ha recibido atención significativa es la educación, dada la suposición fundamental de que existe una relación positiva entre ésta y la decisión del individuo de convertirse en emprendedor con todos los beneficios que ello trae consigo.

Igualmente Duarte \& Tibana (2009) refuerzan la premisa que cuando una sociedad necesita transformarse y lograr mejores condiciones de vida y cimentar propuestas incluyentes en un Estado participativo y en el reconocimiento de los derechos de los ciudadanos, es desde esta perspectiva que adquiere sentido y valor una propuesta de fomento a la Cultura del Emprendimiento, que convoque energías, y avale espacios de creación e innovación, materializados en oportunidades reales para los seres humanos como protagonistas de las propias transformaciones y el mejoramiento de sus contextos.

El sistema educativo es el medio más expedito y eficaz para transmitir, socializar y re-crear la cultura, por lo tanto es desde la "escuela", en sus diferentes niveles, que se puede materializar el propósito transformador, incluyendo dentro de los currículos y planes de estudio la cultura del emprendimiento en el proceso formativo, y desde allí proyectar la generación de oportunidades laborales, la obtención de 
riqueza, a través de las sinergias entre los distintos sectores e instituciones, para consolidar la educación como un derecho y creando las oportunidades para que toda la población se encamine hacia el desarrollo humano (Duarte \& Tibana 2009).

Dehter (2001), planteó en su trabajo "Responsabilidad Social de las Universidades Hispanoamericanas para la Animación de la Cultura Emprendedora Regional”, que las universidades hispanoamericanas no han logrado dar respuesta a las necesidades sociales relacionadas con el desarrollo empresarial y la generación de empleo. Este autor cuestiona si las mismas no se han concentrado demasiado en la formación del conocimiento, y han dejado de lado transferirlo adecuadamente en función de los intereses y necesidades específicas de las regiones.

Frente al reto de generar inclusión social mediante una mayor productividad, más oportunidades de empleo y sobre todo más estrategias para permitir que las ideas de negocios se conviertan en una realidad permanente; primero se debe reconocer que, la mayor dificultad que afrontan los jóvenes en cualquier parte de Colombia es el desconocimiento de las múltiples oportunidades que tienen para ellos un buen número de instituciones públicas y privadas, que como el Fondo Emprender facilitan recursos para la puesta en marcha de nuevas unidades productivas. Igualmente adolecen de una serie de habilidades y de competencias laborales, generales y de emprendimiento que les permita lograr un desarrollo personal y profesional exitoso. Se desconoce a nivel general la elaboración del Plan de Negocios, el cual debe contemplar toda la información necesaria para evaluar un negocio y los lineamientos generales para su puesta en marcha. (Reporte GEM Colombia regiones / Bucaramanga 2010-2011).

Bellamy (1999) argumenta que el tema de la inclusión es el gran desafío que enfrentan las escuelas e instituciones de todo el mundo y Ainscow, M. (2004), confirma que, en países con economías más pobres, la prioridad son los millones de niños que nunca han visto el interior de un aula. Mientras tanto, en los países más prósperos muchos adolescentes egresan de la escuela con calificaciones de discutible mérito, otros son atendidos en diversas modalidades de educación especial segregados de las experiencias educativas comunes y otros sencillamente optan por abandonar sus estudios dado que las lecciones impartidas parecen ser irrelevantes para sus vidas.

Igualmente, en algunos países, la educación inclusiva se percibe como una modalidad destinada a atender a los niños y niñas con discapacidad dentro del ámbito de la educación general (Ainscow, 2004). Pero, se ha generado una dinámica evolutiva en el entorno, orientada hacia la internacionalización del concepto; es así, como la UNESCO (2001) considera cada vez a nivel más amplio este proceso como una transformación que apoya y celebra la diversidad entre todos los discentes.

Las IES al incluir en su currículo la formación emprendedora se convierte en actor directo para el fortalecimiento de competencias en los discentes para reforzar una sociedad garante de generar más 
posibilidades de posiciones laborales y evitar colapsar el sistema productivo vigente que en lugar de generar empleos está limitando las plazas laborales; es en este escenario en donde, el emprendimiento en las IES se convierte en una estrategia de inclusión social y la academia se convierte en actor social para preparar al discente en la búsqueda de mejorar su calidad de vida. En palabras de Formichella, (2004), el actor social que promueve el desarrollo se convierte en un agente de desarrollo y a él le competen diferentes tareas. Por una parte, debe intentar integrar a los diferentes sectores y actores de la sociedad, también debe mediar entre éstos, coordinar información, articular necesidades, recursos, ideas e intentar que exista una fluida comunicación y una fuerte conexión entre ellos. También tiene que articular entre lo económico, lo político y lo social y tomar conciencia de los efectos de estas articulaciones. Además, si bien el actor social no necesariamente está ligado a la toma de decisiones, él puede conectarse con los organismos encargados de ésta y articular las demandas sociales con los mismos, y puede generar las condiciones necesarias para la toma de decisiones en torno a un proyecto.

La ley de emprendimiento referenciada anteriormente, tiene como principios fundamentales, la formación integral del ser humano, fortalecimiento de procesos de trabajo productivo, promover la asociatividad, desarrollar la personalidad y apoyar los procesos de emprendimiento sostenibles de carácter: social, cultural, ambiental, regional e internacional. La Ley pretende promover el espíritu emprendedor, desarrollar y fomentar la cultura emprendedora, que Colombia tenga emprendedores con ideas de negocios exitosas. Crear un vínculo entre el sistema educativo, la empresa, el estado y el emprendimiento.

Generar empresas competidoras y reconocidas en el mercado nacional e internacional. Promover la innovación y la creatividad y Fortalecer las unidades productivas existentes (Ley 1014, 2006). Para lograr la realización de los parámetros anteriormente referidos, el gobierno nacional se ha comprometido a promover y garantizar el vínculo entre el sistema educativo, la empresa y el emprendimiento, designar recursos monetarios públicos, físicos y humanos, a través del ministerio de comercio, industria y turismo, para apoyar las redes de emprendimiento, buscar acuerdos con las instituciones financieras para apoyar a los nuevos emprendedores, generar condiciones para que en las regiones surjan fondos de inversionistas que apoyen las ideas de negocios y establecer fondos a través de los planes semilla y de capital de riesgo para apoyar nuevos emprendedores. Además, junto a las regionales de emprendimiento, como red de emprendimiento nacional que está conformada por quince (15) instituciones públicas y privadas como lo son: Los Ministerios, SENA, Colciencias. FENALCO, ACOPI, Planeación Nacional, las Cajas de Compensación Familiar y otras fundaciones, se busca consolidar el apoyo a la cultura del emprendimiento (Reporte GEM Colombia regiones / Bucaramanga 2010-2011). 


\section{4.- DISCUSIÓN}

Una vez realizado el proceso de revisión bibliográfica se puede determinar que el rol de la Ciencia, la Tecnología, la Innovación (CTI) y ahora el emprendimiento, en la gestión de soluciones a situaciones de pobreza y exclusión social se ha convertido en un tema importante y de seguimiento en el entorno académico, pero, sobre todo, en la perspectiva de las políticas públicas, a nivel nacional e internacional. De este modo; acorde a Thomas, Bortz, Garrido, \& Garrido, (2015), el abordaje de esta temática es clave para repensar de forma integral las políticas de inclusión y desarrollo en cualquier punto geográfico. Para ello, es necesario tomar en cuenta la dimensión tecnológica y cognitiva en los procesos de cambio social y en las estrategias de resolución de las problemáticas de pobreza y mejoramiento de la calidad de vida.

Pero, como iniciar estas propuestas sin poder dar una respuesta clara y oportuna en pro de poder mejorar la calidad de vida de los actores interesados en el proceso frente a posibles interrogantes como: ¿Puede el emprendimiento ayudar a solucionar los problemas de exclusión social? ¿Cuál es la dirección que deberían adoptar las Instituciones de Educación Superior para favorecer estas dinámicas? ¿Cuál es el rol de las empresas, comunidades locales, movimientos sociales y del sector público en estos procesos? ¿Cómo hacer confluir la agenda de I+I+D y las capacidades científicas disponibles hacia problemas locales? ¿Qué políticas públicas y modelos de gestión son los más adecuados para generar procesos de desarrollo tecno-cognitivos que sean participativos, inclusivos y sustentables desde las IES? Y, finalmente, ¿cómo el emprendimiento se convierte en una estrategia de inclusión social en las Instituciones de Educación Superior?

Como se puede apreciar, la cultura del emprendimiento es una estrategia para el desarrollo inclusivo, y para fomentarla, se debe establecer la enseñanza obligatoria del emprendimiento, desde el preescolar hasta la universidad, conformando sistemas de comunicación y orientación profesional en convenio con el SENA, ICFES, COLCIENCIAS y FONDO EMPRENDER. A la par con lo anterior, se deben estructurar formadores emprendedores, con programas de acompañamiento, para la consolidación de empresas a través de actividades como asesorías, consultorías, plan padrino y otras, con programas de comunicación y promoción hacia la cultura emprendedora, que generen espacios para la asociatividad y generación de redes como: Los Prodes (Programas de Desarrollo Empresarial Sectorial) coordinados por ACOPI, alianzas estratégicas entre empresas de un mismo sector económico, las cadenas productivas, los CLUSTER (Concentración geográfica de empresas e instituciones especializadas y complementarias de actividades relacionadas) y defendiendo la cultura del emprendimiento, con estrategias de posicionamiento a través de los medios de comunicación, los centros educativos e Instituciones de Educación Superior con las Unidades de Emprendimiento. 
Revaluando a Díaz \& Celis (2011), cuando afirman que aunque la Ley 1014 dice que "La educación debe incorporar, en su formación teórica y práctica, lo más avanzado de la ciencia y de la técnica, para que el estudiante esté en capacidad de crear su propia empresa, adaptarse a las nuevas tecnologías y al avance de la ciencia, de igual manera debe actuar como emprendedor desde su puesto de trabajo" (Ley 1014 de 2006), lo que se ha venido haciendo es la conformación del área o asignatura de emprendimiento totalmente separada de las áreas de ciencias y tecnología. Ello obedece al poco estímulo que tienen la ciencia y la tecnología en el contexto del sistema educativo colombiano, y al abandono en el que se dejó al equipo docente, quienes tuvieron que asumir el tema del emprendimiento en cumplimiento de la Ley, sin la más mínima capacitación para la comprensión y desarrollo del área; al día de hoy es más diligente el proceso, las IES están orientando recursos tanto físicos como financieros para lograr cumplir con objetivos ya no solo institucionales o por solicitud del MEN sino por necesidad del entorno y de los planes de desarrollo locales, nacionales e internacionales como los objetivos de desarrollo del milenio dados por la Organización de las Naciones Unidas (ONU) a partir del año 2015.

Así como García (2015) lo afirma, las universidades están en permanente transformación, pero ahora se perciben tendencias globales que pueden alterar profundamente el escenario de la educación superior tal como se conoce y que enfrentan a las instituciones universitarias a importantes retos para adaptarse a esos nuevos escenarios y cambios. Se debe entonces iniciar procesos de reorientación sobre las características y previsibles consecuencias de las cinco tendencias siguientes: la renovación de la demanda de enseñanzas, cualificaciones y modelos educativos; el aumento de la oferta educativa y de la educación transnacional y la internacionalización; la consolidación de nuevos esquemas de competencia y cooperación universitaria; la irrupción y disrupción del componente educativo digital; y la transformación de los esquemas de financiación y organización y la necesidad de una gestión eficiente pero enfocados hacia el emprendimiento social.

Frente a esta temática de desarrollo y sobre todo del desarrollo social que conlleve a un escenario de igualdad en el mejoramiento de la calidad de vida de los actores involucrados, Sutz (2010) dice que la desigualdad está en la raíz de las divergencias observables en materia de desarrollo. Entre los factores que con vigor creciente estructuran la desigualdad se encuentran el conocimiento y la innovación, claves para un crecimiento económico que no es hoy menos excluyente que ayer. Conocimiento e innovación podrían, también, convertirse en factores mayores de inclusión social. En este trabajo se argumenta que ello no ocurrirá sólo por el aporte a un crecimiento económico que además mejore la redistribución; tampoco a partir de soluciones muy costosas que se vuelven parcialmente accesibles a través de grandes esfuerzos financieros; se debe hacer ahora más que nunca más social lo social, pensar para el largo tiempo y 
reaccionar en el corto; porque, como lo afirmó el economista ingles Keynes: "En el largo plazo todos estamos muertos" (Keynes, 1936).

Complementariamente, resulta fundamental convocar y movilizar las capacidades de creación de conocimiento y de innovación para resolver problemas que se plantean a nivel de la vida cotidiana y de las necesidades de los sectores más postergados de la población. "Una agenda desde adentro", que oriente la búsqueda de investigadores y de empresarios innovadores; "una agenda desde afuera", que oriente las diversas políticas públicas hacia una fuerte conjugación de conocimiento, innovación e inclusión social; una "agenda desde el margen" que plantee demandas, necesidades, problemas y tipos de soluciones requeridos; los autores comparten esta afirmación de Sutz (2010), porque es precisamente a partir de plantear el trabajo desde agendas que involucren a toda la comunidad que se estructuran iniciativas para proponer como lineamientos e insumos para políticas públicas locales y nacionales.

Hoy en día es habitual que los jóvenes tengan dificultades para encontrar un trabajo, que consigan un empleo temporal o como lo dice Miranda (2008) una changa, que cobren bajos salarios, o estén disconformes con su puesto y quieran cambiar de ocupación. Es frecuente también que frente a estas dificultades -algunos jóvenes- permanezcan por un período más extenso en la educación, se queden muchos años en la casa familiar, retrasen las uniones conyugales y la maternidad. Así como es usual que otros jóvenes- abandonen la educación, se integren tempranamente a ocupaciones del sector informal, encaren embarazos a edades tempranas, o simplemente no hagan nada (es decir, no estudien, ni trabajen). Entre ambas situaciones media una fuerte distancia social que el factor emprendedor podría encaminar y por qué no fortalecer, sobre todo cuando en el mismo camino se le brinda la oportunidad al discente de continuar con sus estudios, pero también de encontrar un medio para generar ingresos y mejorar su calidad de vida.

Igualmente, Kantis, Ishida, \& Komori (2002) presentan un panorama en donde el análisis del desarrollo emprendedor plantea la necesidad de implementar iniciativas que cuenten con la participación de una amplia gama de instituciones, cuya conformación puede variar de acuerdo con las condiciones específicas de cada país. Las universidades deben ser estimuladas y asistidas para que adopten un papel clave en el campo de la promoción del desarrollo emprendedor, tal como se observa a nivel internacional. En América Latina, donde la mayoría de los estudiantes asisten a universidades públicas, los responsables de políticas deben asegurar su compromiso institucional con una estrategia de este tipo.

De lo contrario, el origen social de los nuevos emprendedores estará más restringido a los segmentos medio-altos y altos de la población, los que cuentan con redes sociales más efectivas, con mayor capacidad financiera y con una formación universitaria en instituciones privadas que han tendido a incorporar desde más temprano la promoción de la empresarialidad a sus currículos. Siguiendo un criterio 
similar, el tejido institucional de apoyo a la empresarialidad debe ser amplio y no debe limitarse a las universidades. Esto significa que debe involucrarse a otras instituciones tales como las que trabajan en la formación de adultos, las fundaciones privadas, las cámaras empresariales y otras organizaciones de la sociedad civil que permitan incluir también a la población no universitaria; como se parecía entonces, el camino no es fácil, existen diversas formas para empoderar el sistema pero se requiere de un real apoyo por parte de actores privados como públicos para lograr al menos potenciar un arranque automático y sistémico.

En concordancia con lo afirmado, para que una estrategia de desarrollo emprendedor tenga lugar, los gobiernos (en sus diferentes niveles) deben jugar un papel catalizador: "conceptualizando la estrategia y construyendo la visión, movilizando a los actores clave y comprometiendo recursos para promover la emergencia y desarrollo de nuevos emprendedores y de emprendimientos dinámicos" (Kantis, Ishida \& Komori 2002).

Finalmente, apoyando a González (2013), los profesores, investigadores, alumnos y la comunidad académica, deben ser conscientes, para aportar conocimientos y tiempo, en la elaboración de propuestas y proyectos concretos que impacten el desarrollo de las zonas donde se encuentran insertos, es tiempo de considerar la situación privilegiada de universidades e instituciones de educación superior y el papel que pueden jugar en la elaboración de políticas públicas de desarrollo en las comunidades marginadas. El desarrollo local, regional, nacional e internacional es un tema que se debe considerar prioritario ante el fenómeno de la desigualdad en el país; se recomienda continuar con el seguimiento a los procesos que la ley ha permitido iniciar para lograr que la cultura del emprendimiento en las instituciones de educación superior se convierta en una estrategia real de inclusión social y como se ha podido evidenciar en este trabajo, que el emprendimiento sea más que una estrategia ,una política para mejora la calidad de vida a una sociedad que debe ser inclusiva, social e igualitaria.

\section{5.- REFERENCIAS}

Ainscow, M. (2004). El desarrollo de sistemas educativos inclusivos: ¿Cuáles son las palancas de cambio? Journal of Educational Change, 5(4). Pp. 1-20.

Bellamy, C, (1999) The State of the World's Children: Education. UNICEF.

Blanco, G. (2006). La equidad y la inclusión social: uno de los desafíos de la educación y la escuela hoy. Reice: Revista Electrónica Iberoamericana sobre Calidad, Eficacia y Cambio en Educación.

Camacho, D. (2007). Hacia un modelo de emprendimiento universitario. En Apuntes del CENES, 27 (43), $275-287$. 
Cubillos, M. \& Rodríguez, S. (2013). Una mirada a la docencia del emprendimiento en IES colombianas. Ponencia presentada en: Formando comunidades para el emprendimiento sustentable: VII Workshop Red Emprendesur. Medellín.

Dehter, M. (2001). El ser emprendedor: Modelo para desarrollar comportamiento emprendedor individual y corporativo. Programa Permanente de Capacitación para Dirigentes. IDEB.

Díaz, C. \& Celis, J. (2011). La formación para el trabajo en la educación media en Colombia. Revista Latinoamericana de Ciencias Sociales, Niñez y Juventud, 9(1).

Dickson, P., Solomon, G., \& Weaver, K. (2008). Entrepreneurial selection and success: does education matter? En Journal of Small Business and Enterprise Development, 15 (2), 239-258.

Duarte, T., \& Tibana, M. (2009). Emprendimiento, una opción para el desarrollo. Scientia Et Technica, 3(43). Pp. 326-331.

Durán, S., Parra, M., \& Márceles, V. (2015). Potenciación de habilidades para el desarrollo de emprendedores exitosos en el contexto universitario. Opción, Año 31, No. 77 (2015): 200 - 215.

Durán, S., Fuenmayor, A., Cárdenas, S. \& Hernández, R. (2016). Emprendimiento como proceso de responsabilidad social en instituciones de educación superior en Colombia y Venezuela. En Desarrollo Gerencial Revista de la Facultad de Ciencias Económicas Administrativas y Contables de la Universidad Simón Bolívar-Colombia, 8(2), 58 - 75.

Formichella, M. (2004). El concepto de emprendimiento y su relación con la educación, el empleo y el desarrollo local. Bueno Aires, Argentina.

García, J. (2015). Nuevos escenarios y tendencias universitarias. Revista de investigación educativa, 33(1), 13-26.

GEM Colombia (2011). Reporte GEM Colombia regiones / Bucaramanga 2010-2011. Bogotá: Editorial Universidad del Norte.

González, A. (2013). La universidad como factor de desarrollo local sustentable. Ra Ximhai, 9(1), 65-78.

Jaramillo, C. (2013). Didáctica del emprendimiento en instituciones de educación superior de la ciudad de Medellín. Ponencia presentada en: Formando comunidades para el emprendimiento sustentable: VII Workshop Red Emprendesur. Medellín.

Kantis, H., Ishida, M., \& Komori, M. (2002). Empresarialidad en economías emergentes: Creación y desarrollo de nuevas empresas en América Latina y el Este de Asia. Inter-American Development Bank. 
Keynes, J. M. (1936). Teoría general de la ocupación, el interés y el dinero. México: Fondo de Cultura Económica.

Ley 789 de 2002 (diciembre 27). Colombia, L. (2002). "Por la cual se por la cual se dictan normas para apoyar el empleo y ampliar la protección social y se modifican algunos artículos del Código Sustantivo de Trabajo" disponible en: http://www.camacol.org.co/legal/leyes.L-789-02. html.

Ley 1014 de 2006. Congreso de la República de Colombia (2006). De fomento a la cultura del emprendimiento.

Ministerio de Comercio, Industria y Turismo \& Asociación Colombiana de Universidades -ASCUN. (2014). Estado del arte sobre emprendimiento universitario. Recuperado de: http://www.mincit.gov.co/mipymes/loader.php?1Servicio=Documentos\&lFuncion=verPdf\&id=7931

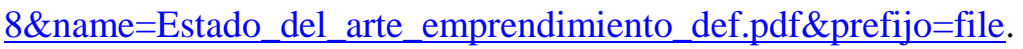

Miranda, A. (2008). Los jóvenes, la educación secundaria y el empleo a principios del siglo XXI. Revista de trabajo, 4(6), 185-198.

OCDE (2001). Schooling for tomorrow. What School for Future? Education and Skills. París.

Organización Internacional del Trabajo - OIT (2004). Tendencias del empleo mundial para los jóvenes. Ginebra: Autor.

Orrego, C. (2009). La fenomenología y el emprendimiento. En Revista Científica Pensamiento y Gestión, 27. Pp. 235-252.

Redondo, R. P., Pertuz, A. D., \& Vidal, J. E. (2015). Modelo de gestión estratégica en unidades de emprendimiento empresarial de la universidad de la Guajira. En Desarrollo Gerencial Revista de la Facultad de Ciencias Económicas, administrativas y contables de la Universidad Simón Bolívar 7(1). Pp. 140-159.

Ramos, F. \& Bayter, L. (2012). Emprendimiento y economía social, oportunidades y efectos en una sociedad en transformación. CIRIEC-Espana, (75), 129.

Reinoso, J. (2008). Experiencias de Emprendimiento en La Universidad del Tolima. Ponencia presentada en el Congreso latinoamericano de Administración de empresas, II Jornadas Nacionales de Innovación y Emprendimiento, realizado en Cochabamba (Bolivia) del 28 al 30 de mayo de 2.008. Recuperado de: file:///C:/Users/db2admin/Downloads/514-984-1-PB.pdf.

Ruiz, M. \& Duarte, T. (2009). Emprendimiento, una opción para el desarrollo. 
Sutz, J. (2010). Ciencia, tecnología, innovación e inclusión social: una agenda urgente para universidades y políticas. Psicología, Conocimiento y Sociedad, 1(01), 3-49.

Tarapuez, E., Osorio, H., \& Botero, J. (2013). Política de emprendimiento en Colombia, 20022010. Estudios Gerenciales, 29(128), 274-283.

Thomas, H.; Bortz, G. \& Garrido, S. (2015), Enfoques y estrategias de desarrollo tecnológico, innovación y políticas públicas para el desarrollo inclusivo, Documento de trabajo IESCT-UNQ $\mathrm{N}^{\circ}$ 1, Bernal: IESCT-UNQ. Disponible en:

http://iesct.unq.edu.ar/images/docs/ThomasBortz-Garrido-Enfoques-y-estrategias-de-IID.pdf.

Toca, C. (2010). Consideraciones para la formación en emprendimiento: explorando nuevos ámbitos y posibilidades. En Estudios Gerenciales. 110 (17). Pp. 41 - 60.

UNESCO (2001). The Open File on Inclusive Education. París: UNESCO. 